\title{
Expression of Spatial Continuity of Architecture and Urban Development within Iranian Bazaars (Case Study: Tabriz Bazaar)
}

\author{
Vahid Mirzaei ${ }^{1}$, Mohammad Reza Bemanian ${ }^{* 2}$, Hamid Reza Saremi ${ }^{3}$, Mohammad \\ Reza Lilian ${ }^{4}$

\begin{abstract}
${ }^{1} \mathrm{PhD}$ student of architecture, Borujerd branch, Islamic Azad University, Borujerd, Iran ${ }^{2} \mathrm{PhD}$ in architecture, Professor, Architecture department, Tarbiat Modarres University, Tehran, Iran ${ }^{3}$ Assistant Professor in Architecture Group, Tarbiat Modares University, Iran

${ }^{4} \mathrm{PhD}$ in Architecture, Assistant Professor in Architecture Group, Borujerd Branch, Islamic Azad University, Borujerd, Iran
\end{abstract}

\begin{abstract}
Architecture is a reality which is recognized within the space. Accordingly, there is a spatial continuity (hierarchy) across various elements in the historical context of old Iranian cities, which had promoted a kind of movement, dynamism, discipline and hierarchy. Without a discontinuity, an individual may find himself/herself within something with the sense of place would be induced into him/her. Iranian bazaars along with adjacent spaces are highlighted examples of such continuity. The present research is mainly aimed at providing a pattern of communication and physical spatial continuity within old Iranian architecture to be used by contemporary architecture. The main research question is that: how can one express the spatial continuity of architecture and urban development across bazaar?

Followed in the present study is a descriptive - analytic approach. In particular, Tabriz Bazaar is considered via data gathered by documentary - field methods. The results emphasized on the fact that, meeting the principle of hierarchy, a spatial continuity is established, i.e. any activity defines a specific scope and territory. Furthermore, communication and physical features (the principle of link among urban elements) of architecture and urban development are created. Working as a joint, the principle of spatial continuity either connects or disconnects a place to/from another place; it is the physical connection between the spaces and their continuity.
\end{abstract}

Keywords: spatial continuity, bazaar, spatial perception, hierarchy

*Author: bemanian@modares.ac.ir 


\section{Introduction}

$\mathrm{T}$ raditional - historical contexts comprise an undeniable reality about the cities with a great deal of historical background. Living not that long ago, a part or area of the city have hosted transportations as well as material and spiritual transactions among the people of that time. In other words, "old urban contexts are a creative living domain within which to understand the position of human within the world and his ties with the past, a place for environment alteration and modification of the view to the present as well as the future" (Habibi, 2011). Possessing evolved features within consecutive centuries, Iranian cities underwent a great transformation within just a few decades. What was referred to as physical characteristics of these cities was a set of valuable architectural - urban development elements arisen from the culture governing the citizens' life. Each architectural - urban space or element has been formed and evolved according to routine needs by the people living in the cities, to meet the requirements of urban society's growth and evolution throughout the time. Old contexts along with their urban elements and spaces such as street network, bazaars and water storages, mosques, inns, etc. not only enjoyed significant physical form, but also had latent physical characteristics as well as special cultural, social and historical values. Started decades age, urban practices and plans have ignorantly provided a basis for urban traditional values to be destroyed. What was referred to as modern urban development arrived as a pervasive wave, first at major Iranian cities, and then at the entire country, was nothing but a reflection of western evolutions. Associated with alterations in the urban life style, years following the industrial revolution in the west were accompanied with influence of colonizers onto Iran when patterns and impressions were taken from western urbanization and urban planning (Nasr, 1986). First introduced in 50s, the identity crises is a still alive subject. The most clear physical presentation of identity crises across historical cities of Iran is seen to be spatial discontinuity of architecture and urban elements in scales ranging from micro to macro.

This is while the Iranian architectural scholar, Nader Ardalan, believes that Iranian architectures have tried to pass the human through an obstacle-free space (vacuum) and not through a solid bulk (plenum); accordingly, "it generates no continued discontinuity or barrier along the human path way. Thu human typically moves forward within a raging and spreading space which is uniquely attached" (Ardalan,2001). In such an urban development, the passage from a closed space to another closed space is successively repeated with no discontinuity ever, because the building is incorporated into a continuous compact framework. The building is not represented as an isolated singular construction; it is not a wanderer component placed on a surface, it is, however, incorporated into the urban context the context to which dominant significant lines including the location of and pathways toward the mosque and other gathering centers, give a dimension and emphasis with the flow directions toward the mosque, bazaar and streets being determined by major and dominant positions and occasions (Stirlen, (1998). Regarding the mentioned issues, spatial continuity and the sense of correlation between Iranian architecture and urban development comprise the main objective followed in this research; it is, however, achievable only with an analysis of communication spaces and elements of spatial continuity between architecture and urban development.

\section{Research Background}

Edward T. Hall believes that the human's perception of space is a dynamic concept, because it is related to an action, i.e. what can be done in a given space, and not to what is seen by the passive insight (Edward, 2013).

Alexander show us the discontinuities within arenas while emphasizing on their continuity: [any internal arena continuity can only be obtained via transition spaces specific to those arena] (Chermayov,1997).

Chermayov suggests that: for any arena, the internal continuity should be maintained with the hierarchy across the arenas being highly 
dependent on the interactions among the arenas (Chermayov,1997).

Christian Nurberg believes that: depending on the time and place, architecture has been presented in various forms with its codes of artistic expression including infinitely many levels. The fundamental language of architect have been applicable visually everywhere, every time, because it reveals the definite and fundamental presence of lifeworld (Norberg Shoultz,2012).

\section{Bazaar}

Bazaar is the most important communication axis and urban space within the old Iranian cities. Based on the existing documents, one may guess historical background of bazaars to root back to several thousand years BC. Until early contemporary, the bazaar in each city was organically connected via a consistent link to other urban spaces, Physical characteristics of permanent covered bazaars were in such a way that provided a pleasant adequate environment for pedestrians and purchasers (Soltan Zadeh, 2014).

In fact, bazaar, as a passing space, creates a domain which incorporates different spaces into each other; i.e. it possess a linear form which makes other cores to interfere with and transform into each other. Just like a number of water droplets placed on a magnetic field which, due to their molecular properties, immediately coalescence, so that no definite border can be considered for any of droplets. Another not to be missed important issue in the spatial structı

bazaar, is that in the Iranian cities, bazaar links the city to micro spaces. it makes "a kind of spatial continuity" to eliminate the sharp boruer between the architecture and urban development; this is what makes bazaar is a dynamic active space of high cultural capabilities and transforms it into a place for social interactions. Bazaar represents the existence of a public place within which not only free and unpretentious footworks are addressed, but also trading affairs are undertaken within various contexts (Diagram 1).

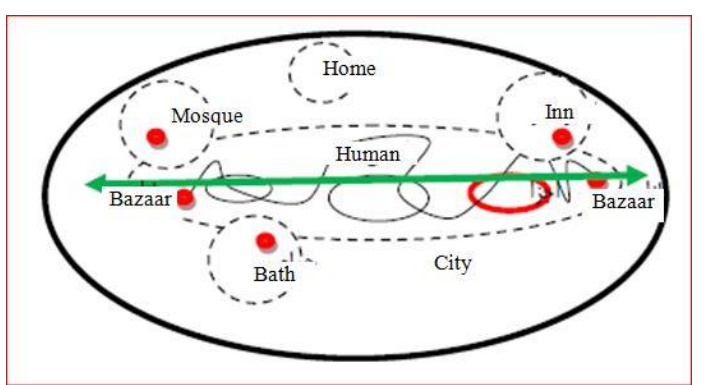

Diagram1: Spatial continuity of old bazaars

(Source: authors).

Spatial continuity is also "one of the fundamental aspects in the old Iranian architecture and urban development. In this type of urban development. The transition from a space into another space is repeated over and over, with this continuity never interrupted. In fact, within such spaces, one may not even need to exit from an existing given environment; instead, a set of well suited passages are provided end utilized (Stirlen, (1998)., i.e. urban spaces are separated based on the passages without losing their "continuity" on the surface, homogeneously developing the city around principal points and groups (such as mosques, squares, etc.).

\section{Spatial Perception}

Normally speaking, perception of a phenomenon, or putting it in better words, the qualities of each phenomenon psychologicallyemotionally affect us - let's say rises an emotion in us or induce us a sense of this or that. For instance, perceiving a phenomenon we begin to have a sense identity or belongingness. It is interesting that when perceiving a phenomenon, we develop not only a sense of its existing state, but also a set of expectations we gain about the phenomenon; in relation with the space, these are called spatial expectations. That is, when uealt with a particular space or place, an individual expects certain feelings to be developed within him/her (Akbari Motlagh, 2003). In each and every civilization, virtually everything has been and still is dependent on the human emotions. An environment whose principle factors have nothing to do with the senses is as unpleasant and ugly as is a society staying away from wisdom (Gidion, 2009).

The space can be classified into 3 classes: geographic space, life space, and architectural space (interior or interstitial) (Diagram 2). In terms of perception, the first space is a subjective space which cannot be directly perceived. 
Life space is a semi-subjective space; some of its attributes are perceivable for us while many others are either recognized via information or not recognized at all. The third space which is objectively perceivable, directly sensed and can be recognized via its defining elements (Yorg Curt, 2011). Space is a main factor in the architecture. Space capture means the ability to see the construction and find the key to understand and recognize it (Memarian, 2012).

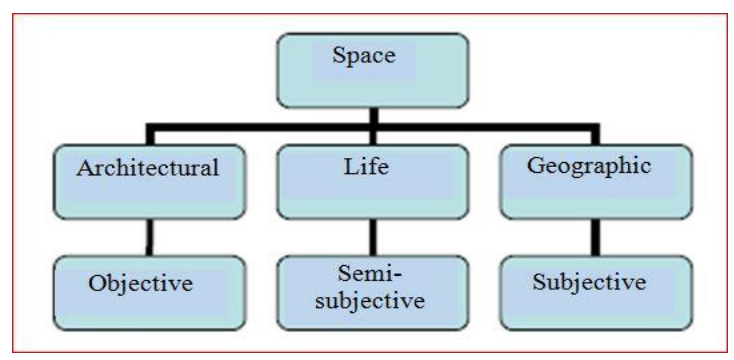

Diagram2: Space classification and space perception approach (Source: authors).

Environmental information is gained via perceptual processes aroused by subjective schema and guided by human needs. Such schema are partly innate and partly learned making a link between perception and cognition. The schema guide not only perceptual process but also sensual (emotional) reactions and actions (spatial behavior); in response, these processes and reactions contribute into subjective schema as a product of perceived behavior. Human senses and actions are created by natural environment capabilities and confined by cultural environment and internal personality of individual (Danesh, 2011). The individual's perception of "social" and "personal" spaces comprise an important aspect of its use of space. Architectural and urban environment created by traditional individuals partly represent such a perception. The space enjoys a principal highlighted position within traditional communities' architecture and design. As important and highlighted are the concepts of time and forum as simultaneous continuities. Space possess active and passive abilities incorporated into every creature. As such, the place, time and forum, the space simultaneously represents its active and passive aspects by motion. Such an integration is observed within urban architecture and environment providing simultaneous motion systems as bazaar creating a continuous stream of harmonic spatial experiences based on numbers and geometries (Traditional Cases). From Stirlen's point of view, spatial perception and understanding of Iranian urban development and architecture is highly dependent on the perception and understanding of spatial continuity and continuation. He sees the resident or consumer as a passenger who has "a continuous sequence of feelings and senses" as he/she is moving from one place to another. "The passengers of this city are constantly seeing themselves within something; they go from one mood to another and from one perception to another; in one place, he/she is immersed into the warm hearty space of the bazaar with just a soft light coming through the filters in the skylight, while in another place...".

\section{Spatial Continuity}

In order to define the term "spatial continuity" we may begin with a look at literal definition of the term "continuity" in various dictionaries.

Continuity. (Hams) the state or the way of being continuous. opposite of aperture. opposite of separation and in-between. opposite of discreteness and dissociation (Dehkhoda, 2nd Edition).

Continuity: the state of continuing for a period of time, without problems, interruptions, or changes (Moein,2015).

In this respect, Ardalan recognizes the spatial continuity as positive spatial continuity:

"Once qualitative aspects of a space are revealed, its qualitative utilities will be immediately emerged. Therefore, the positive essential concept of the space creates all the creatures of the architecture. A positive spatial continuity system creates a sequence of motion systems, connection points, and spatial relations making the promotion and modifications in the metaconscious sense of order possible" (Figure 1).

In total, Iranian architecture and urban spaces are diverse spaces transforming into each other in a compact integrated organization without making any definite borders. The unity of Iranian forms stems from visual interactions of space, shape, and the level of features in each. Indeed, in such urban contexts, the building will not be represented in an individual and isolated 
sense; it is rather incorporated into a compact context in which no discontinuity or discreteness can be found between various elements. Formation of heterogeneous spaces within a continuous body is clearly observable in the spatial structure of Iranian bazaars where each spaces has its own complete structure but they are incorporated into each other as connect to the core of bazaar. Definite borders are vanished and a state of "no border" is established. In bazaar, the connection of a space to another space is inevitably transferred via the principal connection model and achieves the success (Ardalan,2001). (Figure 2). Spatial continuity principle: physical organization of old Iranian cities is based on the spatial link between the elements of the set, e.g. square, main street, city center within the center of neighborhoods. Although exists in the old context of many Iranian cities, spatial community is well clear within the old context of cities located within the deserts. Arranged in an irregular grid, main passages have been making connections among neighborhoods, their centers, and city center complex.

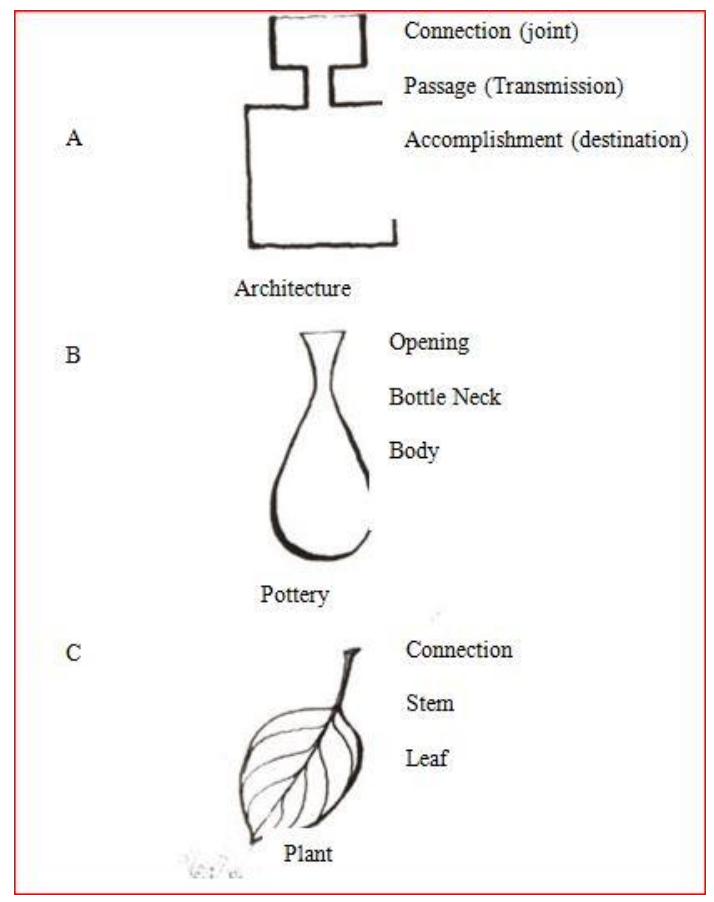

Figure:1 Connection of one space to another following the principle model of connection (Source: Ardalan et al.).

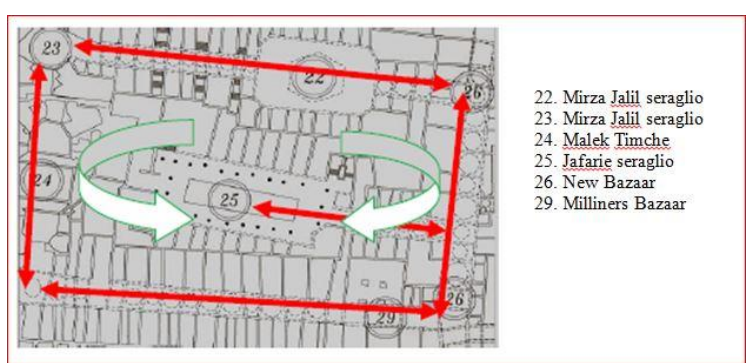

Figure2: Compact integrated organization and connection of one space to another, without generating definite borders, Bazaar of Tabriz (Source: authors).

Furthermore, dividing the space into functional space and transition space can be referred to as another example where the first category includes the main spaces within a building while the second encompasses the transition or connecting spaces. These spaces refer to interstitial or transitional areas. So, in our view of motion and space pass, those participate as a transient space. In addition, there is a relatively more complete classification of spaces in the context of architecture which defines the following three items (Noruzi,2012). (Figure 3):

A. Place space: the main space which embodies a given sense of location or the position of the place,

B. Path space: the main passage spaces defining the orientation of the path. Corridors are examples of such spaces, and

C. Transition space: the auxiliary spaces playing a role in the process of transformation from one situation to another.

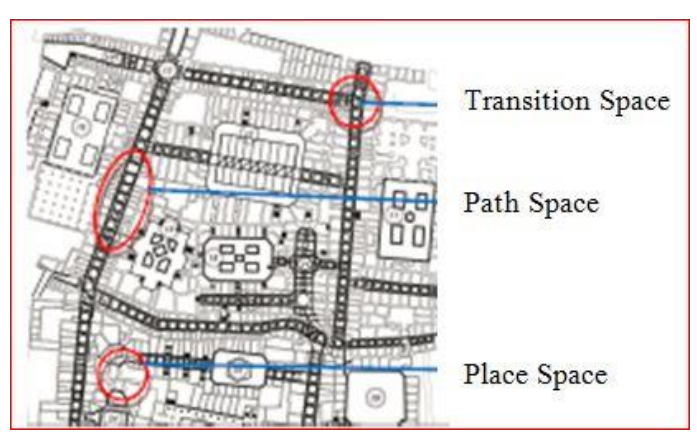

Figure3:Introduction of place, path, and transition spaces within the architectural space of the Bazaar of Tabriz (Source: authors).

In the physical domain, the correlation (which is in fact an indication of differences) is something like a hinge or joint keeping separate elements in a continuous (isolated from each other) form. Jointing can occur for either 
technical (engineering) joints or architectural connecting points; however, these points are usually (though not always) coincide. In architecture, the joints are always acting as a mediator between two ranges thought to be opposite or in contradiction with respect to each other.

\section{Hierarchy}

The principle of hierarchy indicates that there are real differences in the forms and spaces within the majority or even all architectural combinations. Such differences reflect the importance of such forms and spaces as well as the functional, conventional symbolic roles they play within the organization (Ching,2011). Whenever a number of elements are placed together, a kind of discipline is established across their relationships. They may all be of the same value or follow a hierarchy. In architecture, a hierarchy can be considered from multiple points of view. In general, visual hierarchies can be distinguished from spiritual ones. Visual factors include the size, form, and position. The differences in the values of spaces come from spiritual factors (Diagram 3) such as the symbolic significance of an element, or cultural or even personal criteria of particular values (Yorg Curt, 2011).

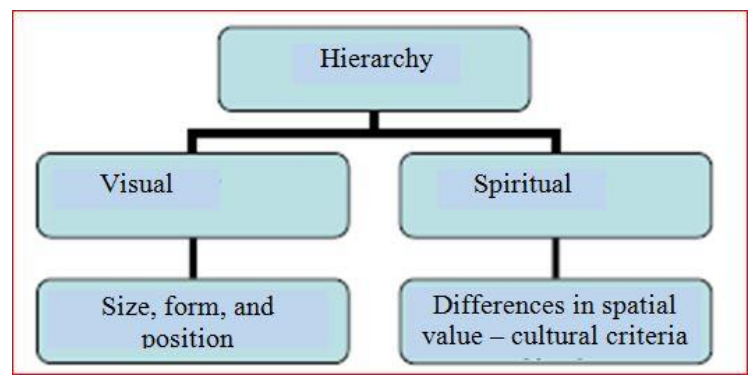

Diagram3:. Different views on hierarchy within the context of architecture (Source: authors).

Hierarchy is among fundamental principles governing the complexes, components and phenomena which either naturally exist as a totality within the universe, or are designed and created by human. The principle of hierarchy plays an important role in defining the components and totality of a complex while gives it an identity. In other words, this is among the criteria which can undertake a fundamental role in defining the discipline governing the complexes and the relationships among their components while being the association of the criteria used to define the coordination of each component. Therefore, features of the hierarchies governing the complexes can serve as an important tool in the course of studies defining and determining the position and value of each component as well as the entire complex, in order to express their features and functionalities, so as to determine the coordination of relationships among the components, the totality and the roles and functions of each one (Naghi Zadeh, 2005). Having been an element of Iranian - Islamic cities, Bazaar is in particularly good position for expressing different aspects of hierarchies within Iranian - Islamic cities. It consisted the most important communication and public spaces within the cities within which the highest volume of footwork was realized. Many other public urban spaces were used to be built next to or near the main bazaar. For example, one may refer to the Grand Mosque which, unless in exceptional cases, was normally constructed next to the bazaar (Soltan Zadeh, 2014). (Figure 4).

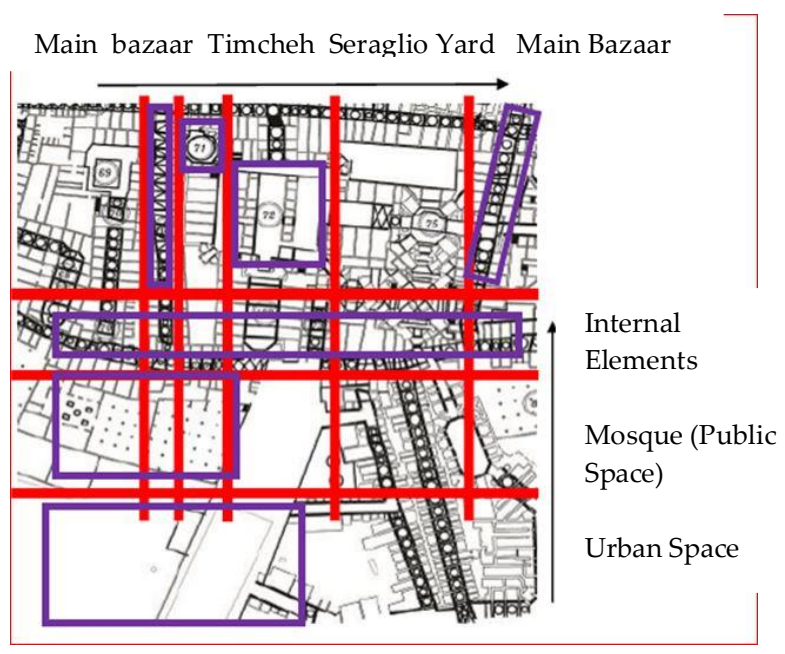

Figure4: Hierarchy of public connecting elements along with internal elements of the Bazaar of Tabriz (Source: authors) - Field survey.

Reported in Table 1 is a spatial and network analysis of the Bazaar of Tabriz according to the investigations performed: 
Table1: Spatial and network analysis of Iranian

Bazaar - The Bazaar of Tabriz (Source: authors).

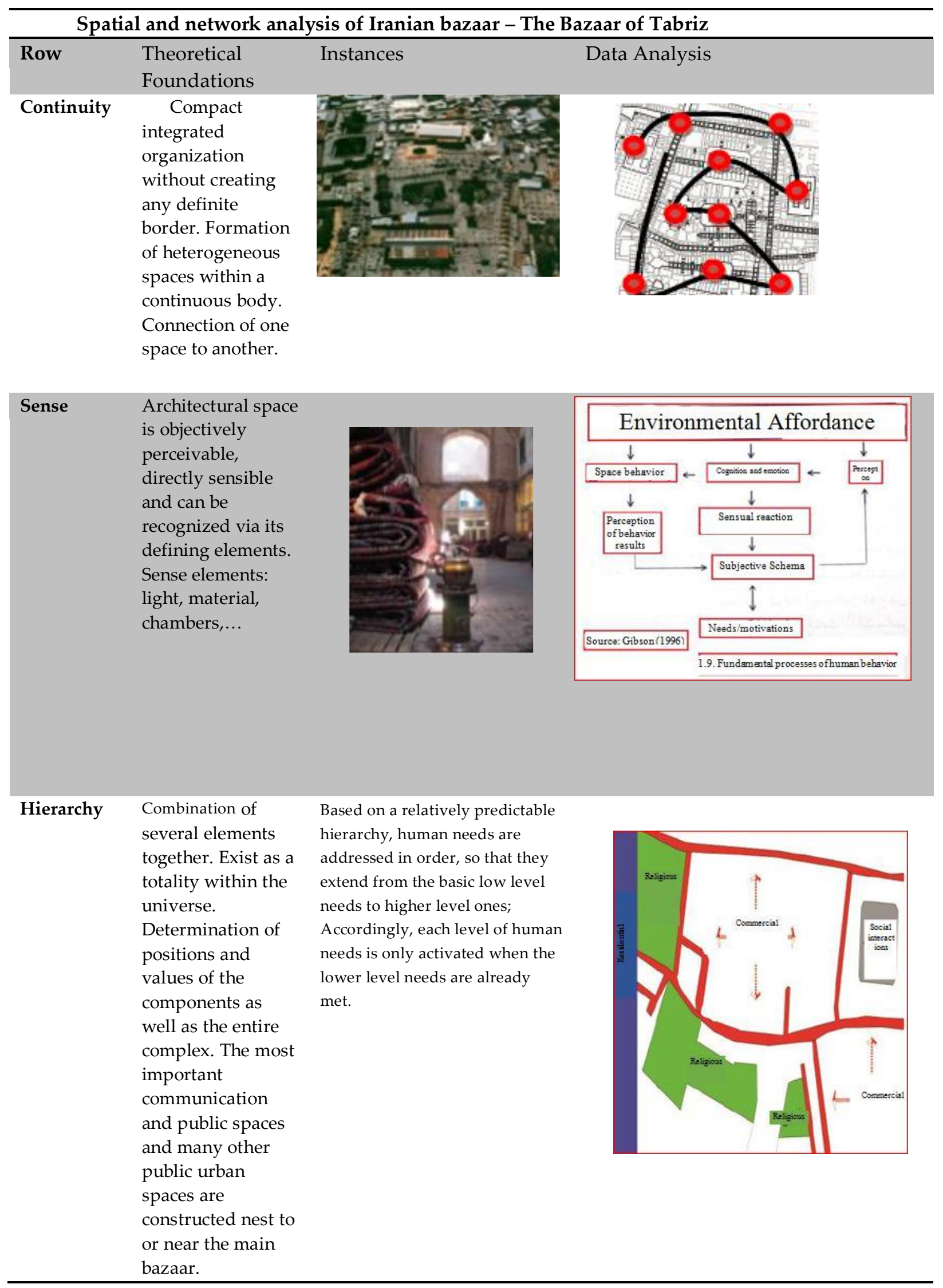




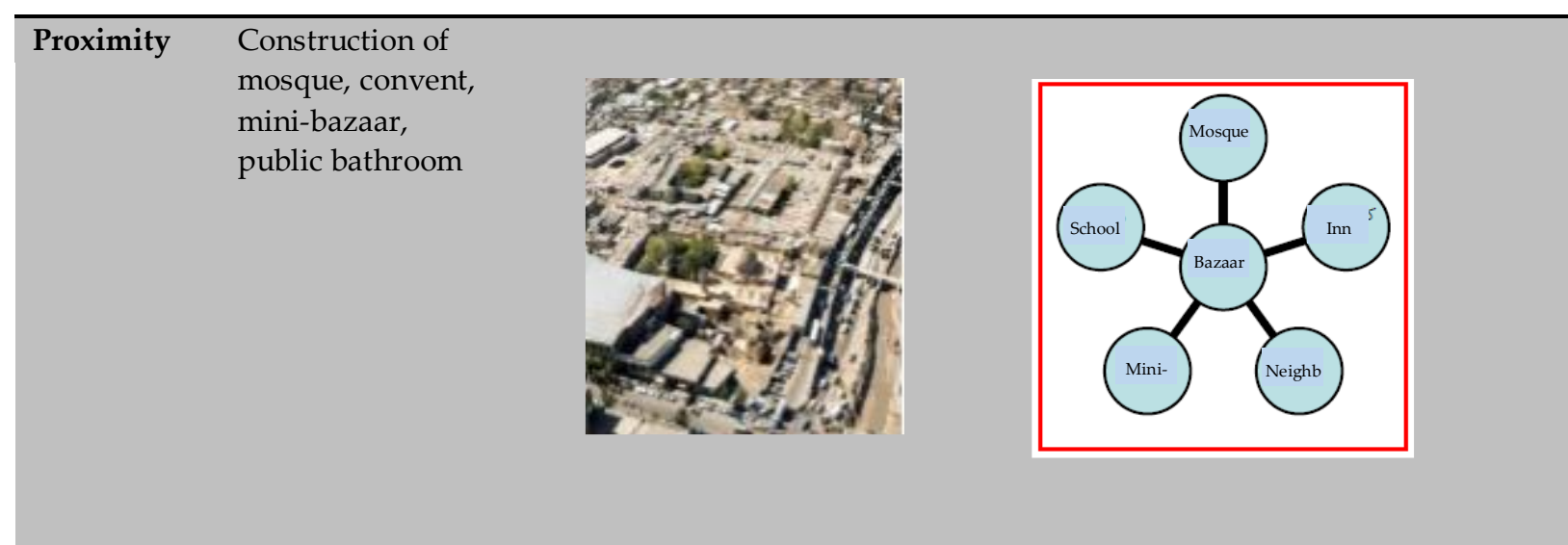

\section{Social In the Iranian}

Interactions cities, bazaar links the city with a

smaller scale

space. It provides

"a kind of spatial

continuity"

eliminates the

definite border

between the

architecture and

the city; this makes
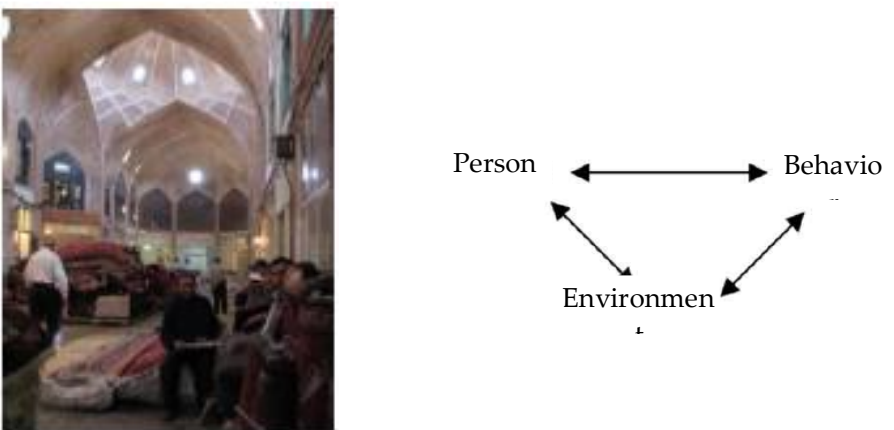

bazaar a dynamic

active place of very

high social

capabilities - a

place for social

interactions.

Religious

ceremonies - News

broadcast -

Mourning events

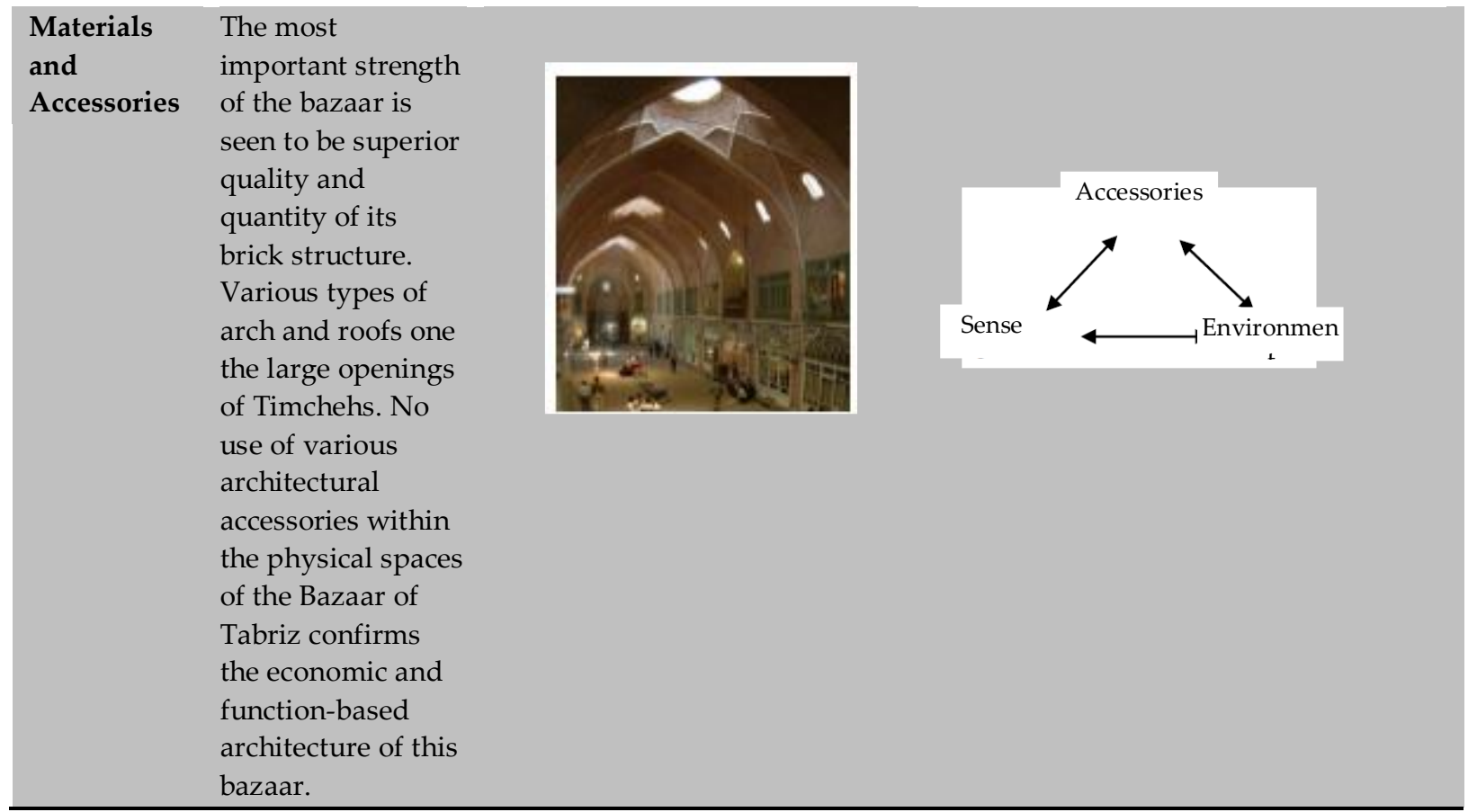




\section{Conclusions}

According to the investigations performed in this research, all elements of bazaar, within the Iranian architectural context, follow a line of discipline and serve each other closely, just like different members of a body, with nothing being negative or excessive. The repetition gives rise to the continuity of the sense of place. While moving in a covered space, one enjoys a sense of security and once he/she passed through the main bazaar, he would come to such spaces as Grand Mosque, inns, public bathrooms, and residential neighborhoods; he/she may further follow the pass through narrow - sometimes covered - alleys to the private space, i.e. the Iranian home with its yard being at the center of sense. Such a motion across different spaces have established spatial and communication hierarchies. This principle is clearly observable in our old cities. However, the hierarchy, the continuity and the sense of presence in the place are among those concepts missed within the current architecture of our country. Therefore, this principle can be used in the current architecture. For example, once passed through the main bazaar, we get to four-leads from where we can get access to other branches of bazaar or the neighborhood, inn, Grand Mosque, etc. (Figure 5). Such a spatial connective principle is completely sensible in terms of physical, meaning and communication aspects; it can be recognized and utilized in the modern architecture in scales ranging from macro to micro.

The attribute of Iranian urban spaces is the physical connectivity and continuity of spaces together. Spatial continuity seems to be established via the principle of hierarchy; that is, each activity defines an area or territory. Furthermore, created via connective and physical features (connective principle of urban elements) are the architecture and urban development.

Creation of such spaces prevents the spaces from being boring or monotonic. Therefore, the principle of continuity acts as a joint to connect one place to another. While being applicable, the spatial performance is defined and visual connection between the architecture and urban space is formed. Hence, the same level of sense of spatial continuation has been induced into the resident. One may suggest mentioned joint between the architecture and urban development to be missed today. As a final word, the solution that can be proposed for the sake of modern age is particularly the connection between the architecture and the city. Is that anything but true expression of spatial elements and concepts, a connection toward a kind of wise awareness of history, humanity and society in its time?

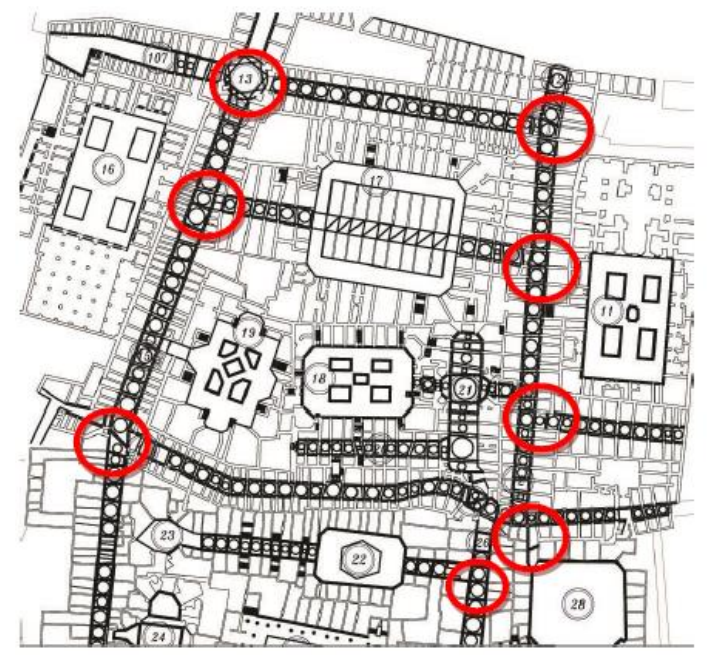

11. Haj Safar Ali mosque

12. Yamanduz Bazaar

13. Great Dalaleduz branch

14. Small Dalaleduz branch

15. Sadeghie Bazaar

16. Sadeghie school yard

17. Shazde Bozorg seraglio

18. Mirza Shafi seraglio

19. Shahzade Koochak

20. Mirza Shafi corridor

22. Mirza Jalil seraglio

23. Mirza Jalil seraglio

24. Malek Timche

25. Jafarie seraglio

26. New Bazaar

28. Darb Abbasi seraglio

107. Yakhchal Sadeghie

Figure. 5. Division of bazaar based on fourleads (Source: authors).

\section{References}

Akbari Motlagh, Mostafa (2003) The Human and Perception of Surrounding Environment, Specialized Quarterly of Architecture and Urban Development, Vol. 70 - 71. 
Ardalan, Nader; Bakhtiar, Laleh (2001) The Sense of Unity, Elm-e-Memari Pub., Tehran.

Ardalan, Nader; Bakhtiar, Laleh (2001) The Sense of Unity, Translated by: Shahrokh, Hamid, Khak Pub., Tehran.

Chermayov, Serg, Alexander, Christopher (1997), Arenas of Collective Life and Private Life, Translated by: Manuchehr Mazini, 3rd edition, University of Tehran Pub., Tehran.

Ching, Francis D. K. (2011) Architecture, Form, Space and Descipline, Trnaslated by: Mahmoodi, Kurosh; Basiri Mojdehi, Reza; 4th Edition, Shahr-e-Ab Pub., Tehran.

Danesh, Jaber; Tabibi, Amir (2011) Presence Quality within Urban Fields with an Emphasis on Iranian Traditional Cases, The Quarterly on Iranian - Islamic Urban Studies, 1st year, Vol. 4.

Dehkhoda, Ali Akbar, The Dictionary of Dehkhoda, 2nd Edition, University of Tehran Pub., Tehran.

Edward T. Hall (2013), The Hidden Dimension, Translated by: Tabibian, Manuchehr. 7th edition, University of Tehran Pub., Tehran.

Gidion, Zigfrid (2009), Space, Time, and Architecture, Translated by: Mazini, Manuchehr, 12th edition, Elmi-Farhangi Pub., Tehran.

Habibi, Seyyed Mohsen (2011), Explanation of Intellectual Currents within Contemporary Iranian Architecture and Urban Development, 2nd Edition, Office for Cultural Researches, Tehran.

Memarian, Gholamhossein (2012) A Plunge into Theoritical Foundations of Architecture, 7th Edition, Sorush-o-Danesh Pub., Tehran.

Moein, Mohammad (2005) Persian Dictionary, Adna, Rah-e-No Books, Tehran.

Naghi Zadeh, M (2005) Foundations of Religious Art within Islamic Culture, Office for Islamic Culture Pub., Tehran.

Nasr, Seyyed Hossein (1986), Sadr-al-Din Shirazi (Mollasadra), Compiled by: M. M. Sharif, History of Islamic Philosophy, IUP, Tehran.

Norberg Shoultz, Christian (2012) Architecture: Presence, Language, and Place. Translated by:
Seyyed Ahmadian, Alireza, 3rd edition, Niloufar Pub., Tehran.

Noruzi, Reza (2012) Conceptual Investigation of "Joint" within Physical Organization of Architecture, Bulletin of Vernacular City and Architecture, Vol. 3.

Soltan Zadeh, Hossein (2014) Iranian Bazaars, 5th edition, Office for Cultural Researches, Tehran.

Stirlen, Hanry (1998) Isfahan, an Image of the Heaven, Translated by: Arjmand, Jamshid, Foruzan Pub., Tehran.

Yorg Curt Gruter (2011), Aesthetics and Architecture, Translated by: Pakzad, J., Homayoon, A., 6th edition, Shahid Beheshti University Pub., Tehran. 